\title{
Performance Analysis of Routing Protocols RIP, EIGRP, OSPF and IGRP using Networks connector \\ $1^{\text {st }}$ AWAN NAHEL MAHMOOD ${ }^{1}$ \\ \{awannahel@yahoo.com\} \\ Assistant Lecturer Department of Control system \& collage \\ Engineering Electronics, Ninevah University, Mosul, Iraq
}

\begin{abstract}
Recently, the Internet relies on a core suite of IP routing protocols. This paper examines the characteristics of important routing protocols in a performance network. Any router in the network needs help with routing protocols. Those deferred types of routing protocols used in networks such as Interior Gateway Routing Protocol (IGRP), Open Shortest Path First (OSPF), Enhanced Interior Gateway Routing Protocol (EIGRP), and Routing Information Protocol (RIP). All transmitted data through the internet between the source and the destination need to be routed using one of the routing protocols. Choosing the appropriate routing protocol by scanning protocols depends on the type of network applications and a number of parameters (i.e. traffic sent and traffic received). In addition, this paper presents advances in routing technology that highlight the difference between RIP, OSPF, IGRP and EIGRP in terms of metrics and other technical aspects. In this survey, networks rely on different types of routing protocols and studies the packets end to end delay, convergence time on flapping links and the average point to point throughput (bit/sec).
\end{abstract}

Keywords: OPNET modeler14.5, RIP, EIGRP, OSPF, IGRIP

\section{Introduction}

Development technology is currently based on IP routing protocols. Data transmitted over the Internet should be routed between networks using routing algorithms. The routing algorithms depend on different parameters for getting the most suitable path selection for transmitting information over the internet (i.e. Bandwidth, cost, packet delay, hop count, and maximum transmission unit). The routing table is used by the routing protocols to maintain the matrices' results. Depending on if the routing is between autonomous systems or through an autonomous system, then two different samples of routing protocols can be selected: Exterior Gateway Protocol (EGP) and Interior Gateway Protocols (IGPs). EIGRP, OSPF, and RIP are commonly used IGPs. Border Gate Protocol (BGP) is a symmetric model to (EGP) is based on routing protocols and created to choose the most suitable path selection through the internet [1-2].

In this paper, some routing protocols like EIGRP, RTP, OSPF and IGRP are proposed to determine the best path using some parameters to determine network performance. The classification of routing protocols is shown in the diagram below. These dynamic routing protocols can be used to configure routing tables in the routers (such as IGRP, RIP, EIGRP, OSPF, and Intermediate System - Intermediate System (ISIS). Exterior Gateway Protocol (EGP) can be used for the routers in different domain networks (i.e. Border Gateway Protocol (BGP)). While; the routing protocols for the routers in the same domain network can be categorized into a static routing protocol and dynamic routing protocol. Both of EIGRP and OSPF are dynamic routing protocols that can be used in both IPV4 and IPV6 as shown in Figure 1. [3] 


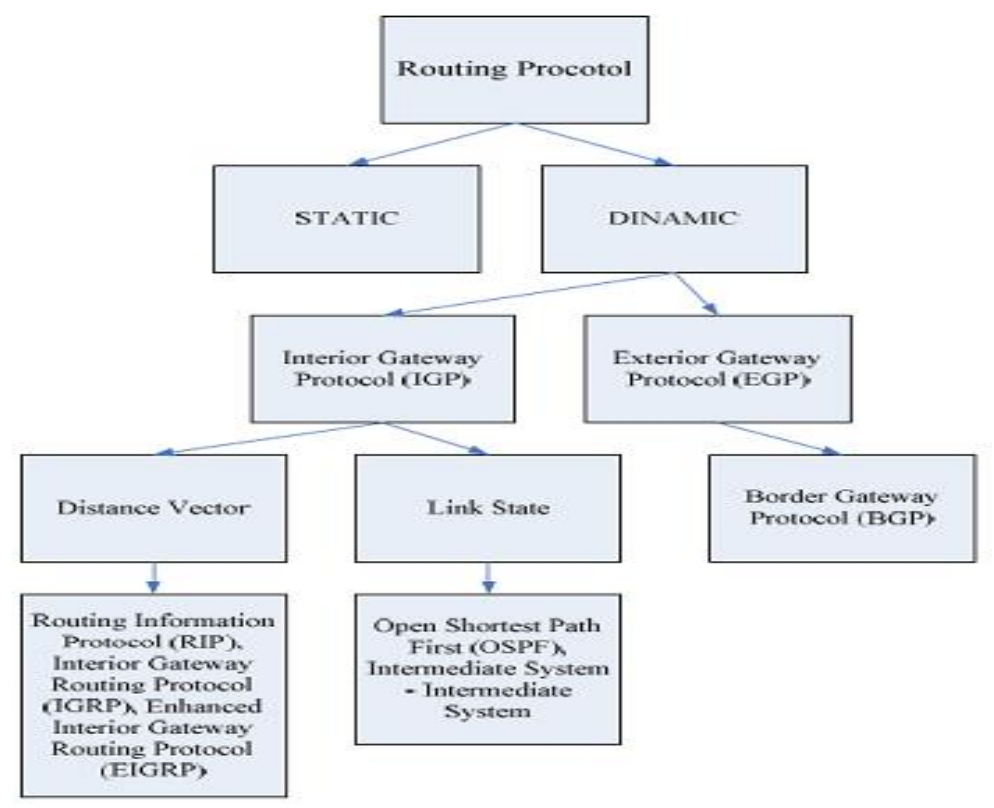

Fig.1. Classification routing protocol [3].

\section{Types of routing protocols}

\subsection{RIP (Routing Information protocol)}

$\mathrm{RIP}$ is the routing protocol of a distance-vector as a metric (using hop count). Its traffic stabilizes and quickly sends the packet transmission over another connection after ensuring that the network is low level. In order to find positive results; the researchers (Fulkerson, 1962, and Bellman, 1957) proposed to update the routing table by each router periodically. Each router sends all the radical information in the routing table to the adjacent routers. In order to update the routing table in each router; when the distance vector receives the data from the adjacent router, it will look for the changes from the previous distance vector. For this reason, the results improved the routing timers and routing update process. Topology updates its routing table with timers. The advantages of static routing using RIP are simple, but updates are not manually updated when the structure changes. [4-8]

\subsection{Enhanced Interior Gateway Routing Protocol (EIGRP)}

IGRP protocol was found in 1992, which was enhanced by a distance vector. IGRP and EIGRP are configured on Cisco routers and have Cisco proprietary. EIGRP can dynamically detect routers connected directly to the network. It is often the same as "Hello" (protocol) that wants to find the near on the Internet. The basic tools for EIGRP for packages sent using "hello" are a similar tool to confirm that each router is working properly. As a support for the network; packet exchanged in a path of high bandwidth is managed every 5 -second time period. In this case of connections requiring low packets, bandwidth, are exchanged every one minute. EIGRP no longer remembers the tours and the selected paths regarding temporary updates in the tradition of convergence topology. Enhanced Interior Gateway Routing Protocol doesn't depend on periodic updates because convergence in topology, but build schame with ads for near about changes in the topology. The data is present as in protocols for a distance-vector. The structural schame is processed to determine the most suitable way for each destination network. Enhanced Interior Gateway Routing Protocol implements an algorithm named as (DUAL) Deployment Update Algorithm. [7-9] 


\subsection{Open Shortest Path First (OSPF)}

OSPF is an Internet Engineering Task Force (IETF) case development by the IGP working group. This group was established in 1988 to design IGP based on the first-most-short-path algorithm used on the Internet and an algorithm based on country links. [5]

Each router connects to another router near it, selective flooding will send these packets where they exchange, transferred to the destination router, and updated their routing table with internal synchronization performance every 30 minutes. Each router maintains its routing table by developing its network drawing database. As in routers dealing with this protocol, information links accumulate in the case by computing the nearest path to a specific network algorithm known as the Dijkstra algorithm. Every node is classified as the distance from the source node to it, and all nodes will be labeled infinite, with all tags initially temporary. [10-12]

(Open Shortest Path First) keep the routing table for every connection in the network but (Routing Information Protocol) keep the routing table for the most suitable route in each goal. Each (Open Shortest Path First) router depots LAN mode with (LSA) Link State Advertisement and broadcast the entire AS. Every (LSA) router created by all routers receives an AS. The Link State Database (LSDB) is formed by the LSA group. Evey (LSA) is a description of the router's perimeter network topology. Thus, LSDB is the reflection of the AS network topology. [13]

\subsection{Interior Gateway Routing Protocol (IGRP)}

IGRP is an IGP distance vector that routers use to exchange routing data within AS. IGRP was partially generated to defeat RIP restrictions (i.e. Single Routing Scale and Maximum Hop Count of only 15) when deployed inside big networks.

Interior Gateway Routing Protocol provides various metrics for each path (i.e. delay, load, reliability, and bandwidth). In order to contrast two paths, multiple metrics are integrated together into one scale, using a formula that can be set using predefined constants. By default, the IGRP is a set of sector-delay metrics and minimum segment bandwidth (i.e. the maximum Hop Count up to 255 (default 100) and routing updates are advertised each 1.5 minutes). Protocol number 9 is reserved for IGRP in communication networks. Since IGRP is a classless protocol that does not contain a subnet mask field, the router assumes that all subnet addresses in the smaller Class A,B and $\mathrm{C}$ network have the same subnet mask. This not applicable to classless routing protocols that can use different length subnet masks. Classless protocols are becoming minimal common because their dissipate (IP) as address space [7].

\section{SIMULATION NETWORK RESULTS:}

Table 1. Simulation Scenario for Network.

\begin{tabular}{|c|c|}
\hline Attributes & value \\
\hline Simulator & OPNET( Modeler 14.5) \\
\hline Type of Router & CISCO 7000 \\
\hline Scalability & (10 x 10) Km2 \\
\hline Traffic type & Voice over IP Call Quality GSM \\
\hline Number of Subnet & 100BaseT_LAN \\
\hline Model object & Time (200 minutes) \\
\hline Simulation &
\end{tabular}

In Table 1, the network consists of 5 subnets connected via a Point to Point Protocol using digital signal 3. Every subnet is composed of 3600 switches Cisco, 7200 routers Cisco, and Ethernet server. Every device are connected to 100BaseT cables Ethernet as shown in Figure 2. This paper proposes a set of routing protocols (such as RIP, EIGRP, OSPF, and IGRP) and a comparative performance analysis of multiple routing protocols based on simulation using OPNET 14.5 simulator in a specific network with semi-network topology. Given the increasing demand for high-speed Internet and the increase in traffic in telecommunications networks, investigations need to resolve bottlenecks that occur in telecommunications networks. In this network, various parameters such as packet delay variation, traffic 
sent, throughput, and end to end delay were examined. In addition to using the application type, for example, Voice over Internet Protocol (VOIP) studies all network conditions. In this paper, simulations are divided into a number of scenarios based on OPNET modeler 14.5.

SCENARIO 1: In this scenario network design Figure 1 consist of a number of internal networks depending on central subnetwork but other together connected to a server in addition study different statement using the type of routing protocols.RIP.

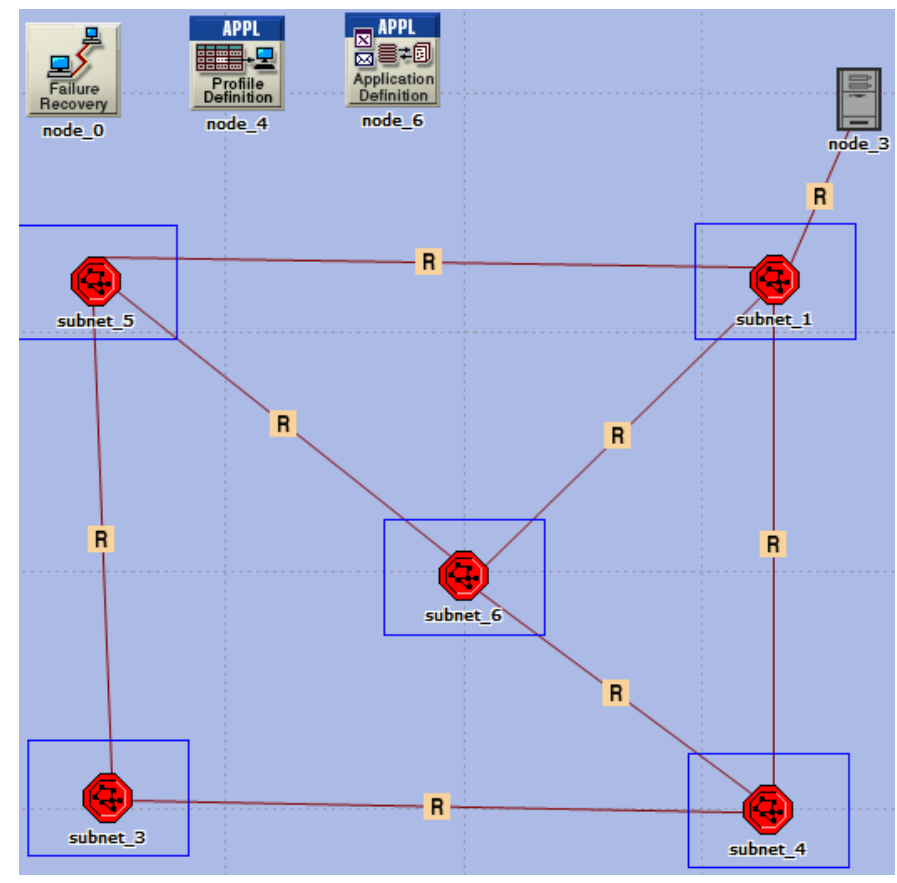

Fig.2. network content on number sub networks used protocol RIP

Figure .2 From this figure study states protocols in network original A schematic view of the internal network contain from fixed node, Cisco 6000 routers and server In this network depend on protocol RIP with application voice over IP with using time running 200 second .

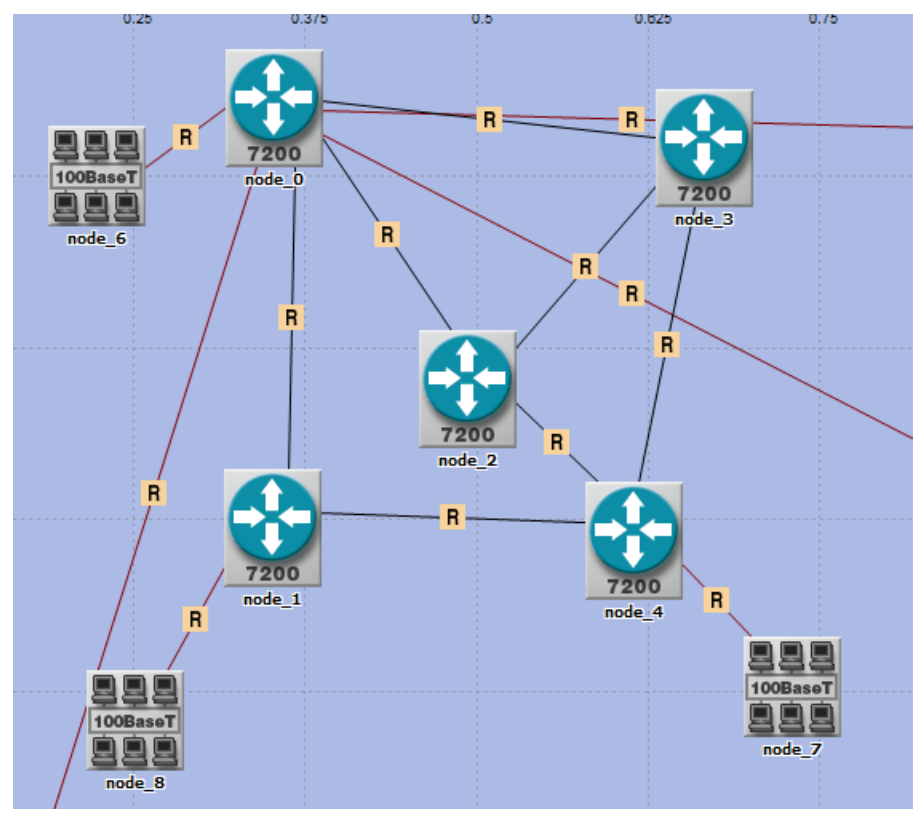

Fig.3. Shown content network internal 
In the internal network consist of router Cisco from type 7200 contact together with groups of Workstations, we applied the Links with transmission rates of $100 \mathrm{Mbps}$, which is called 100BaseT in the software library from OPNET 14.5. For voice application type voice over IP, encoder scheme (G.711silence) addition service interactive voice(5), GSM Quality speech scheme GSM FR that voice and service Best Effort.[9]

SCENARIO 2: In this scenario using the same network in analysis protocols where used protocol EIGRP shown down in Figure 3In this network depends on protocol EIGRP with application voice over IP using time running 200 seconds.

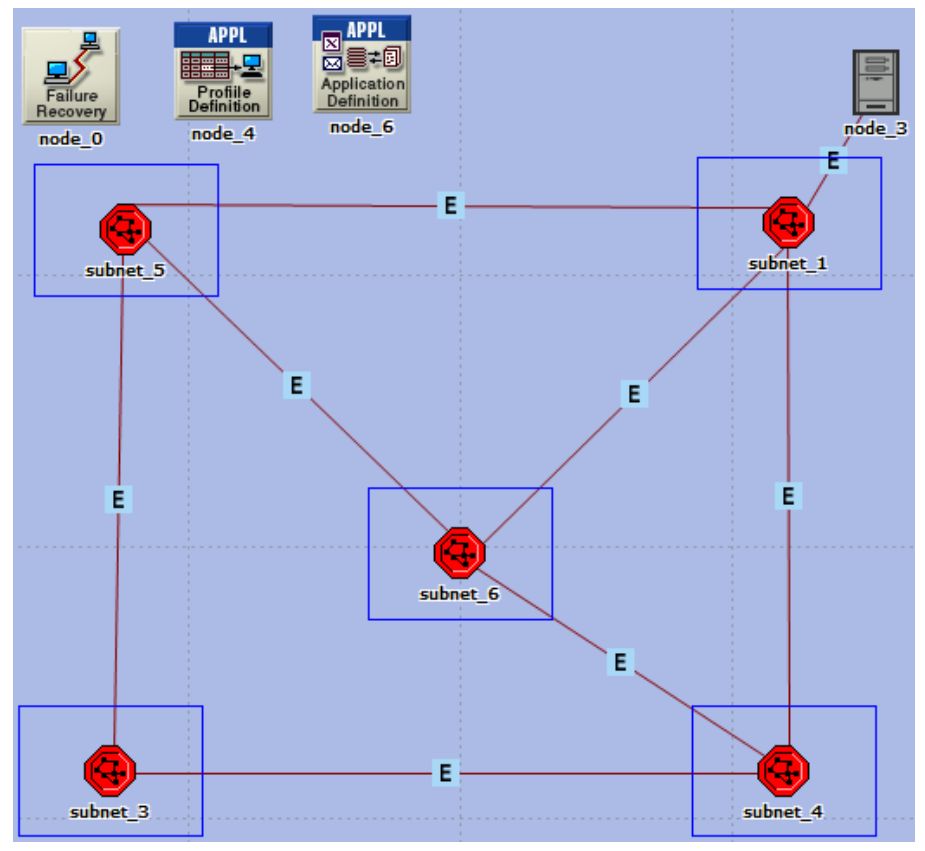

Fig .4. network content on number sub networks used protocol EIGRP

Used voice application (VOIP) voice over IP,Encoder scheme (G.711silence) addition service (5)interactive voice in state used protocol EIGRP.

SCENARIO 3: In this scenario repeat process in same network but another protocol OSPF in addition study performance this protocol from used different parameters shown in internal networks later shown in Figure 4.

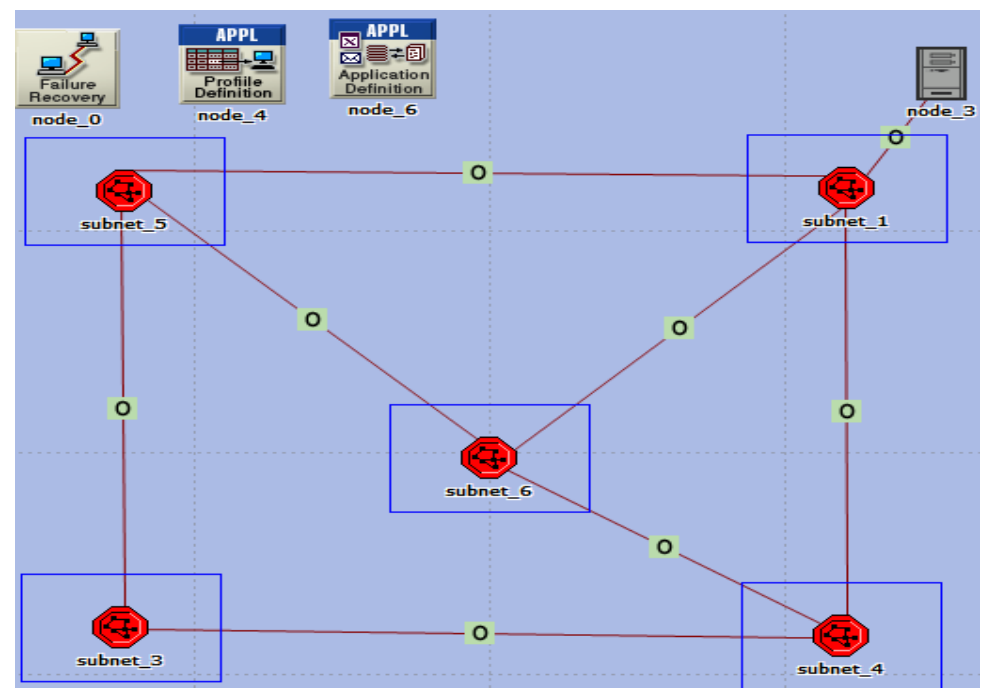

Fig.5. network content on number sub networks used protocol OSPF 
Used 2 type applications from voice over IP, scheme G.711(silence) and voice GSM Quality speech. Through the simulation process, one can get on the traffic sent based on the proposed protocols to differentiate between the levels and the characteristics of protocols on the network. It is found that EIGRP protocol could reach a higher level comparing with other protocols whereas RIP on a low level.

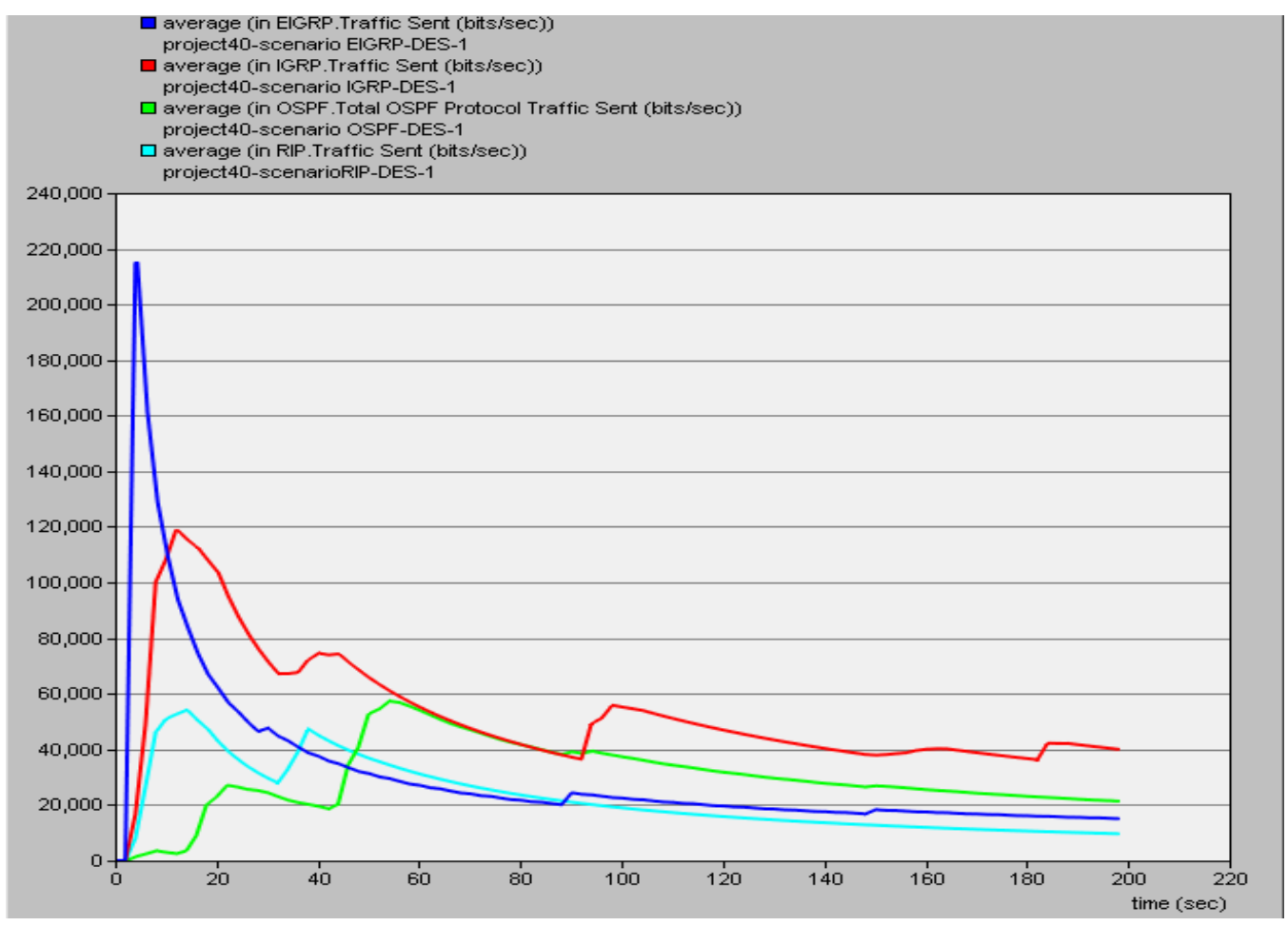

Fig. 6. Shown protocols Traffic sent( bit/sec) on network

From Figure 6 Notice from traffic sent protocols that EIGRP get on high level reach 215,349.34 average traffic sent with time 4 s comparing with RIP to reach 54.168 average traffic sent with time 14 s protocols in network measurement unit data on time, in the same time get on low-level protocol RIP depending on move data from point to other points. 


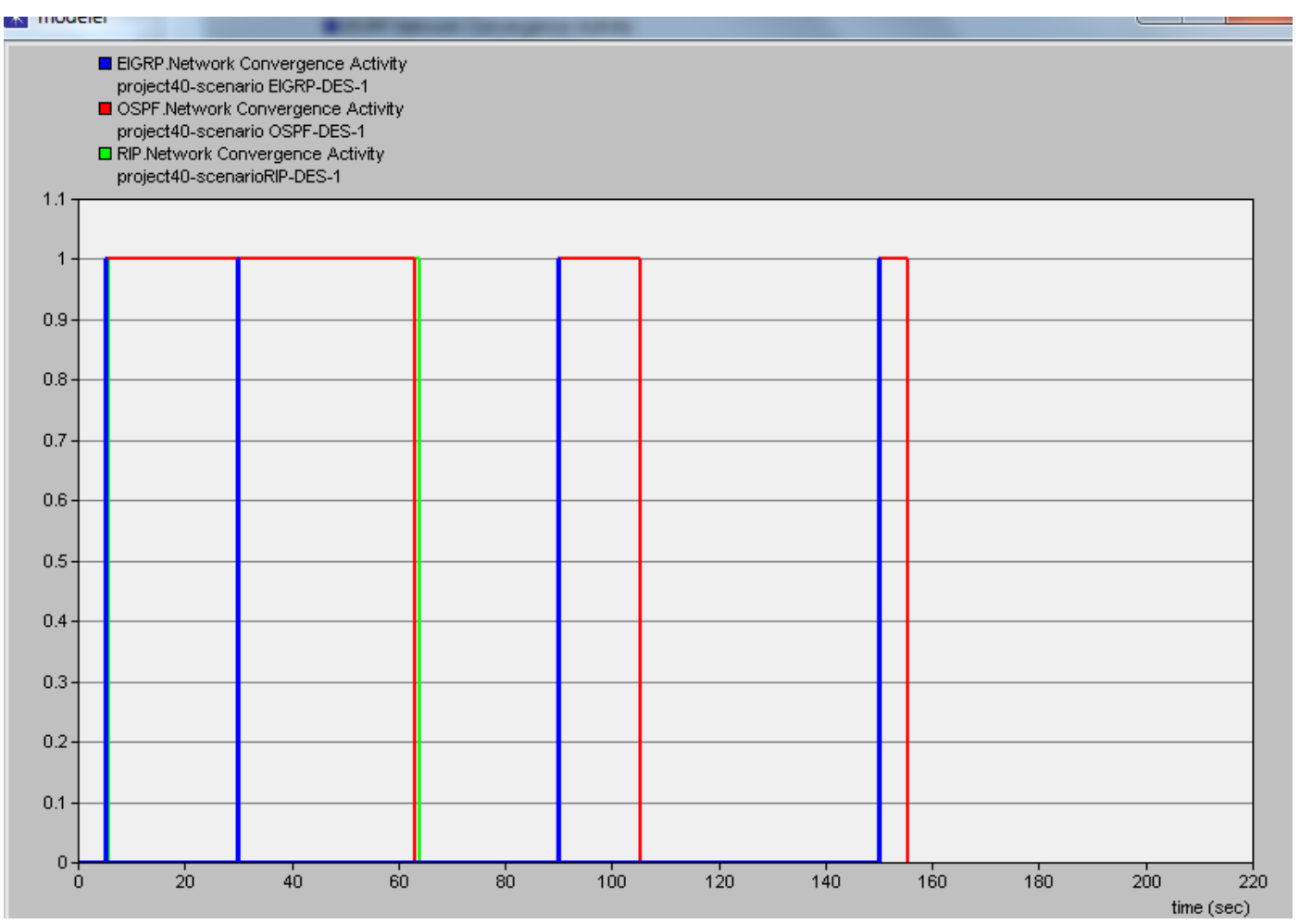

Fig.7. Network convergence activity recovery and over $200 \mathrm{sec}$.

OSPF, EIGRP and RIP protocols represent the lowest and tallest network convergence times, respectively, notice routing protocol OSPF that needs time $155 \mathrm{~s}$ according to the configuration of time summation, whereas EIGRP needs 150 s process time.

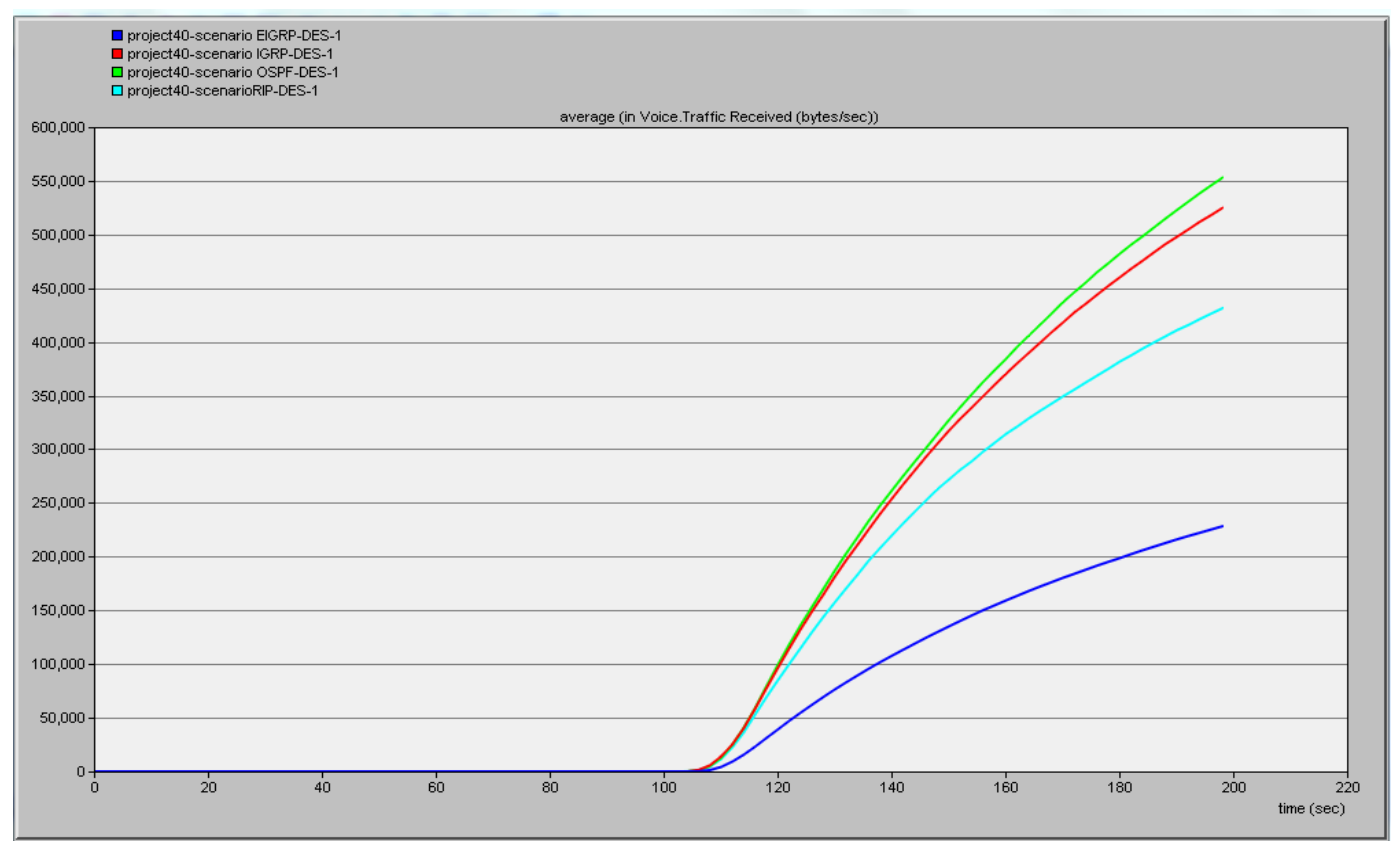

Fig.8. Shown average voice traffic recived (byte on sec ) 
In figure 8. From traffic, result received data (voice ) in unit byte on sec reach protocol OSPF on a high level and protocol EIGRP on a low level in the network .efficiency protocol OSPF is better than protocol EIGRP in traffic received data.

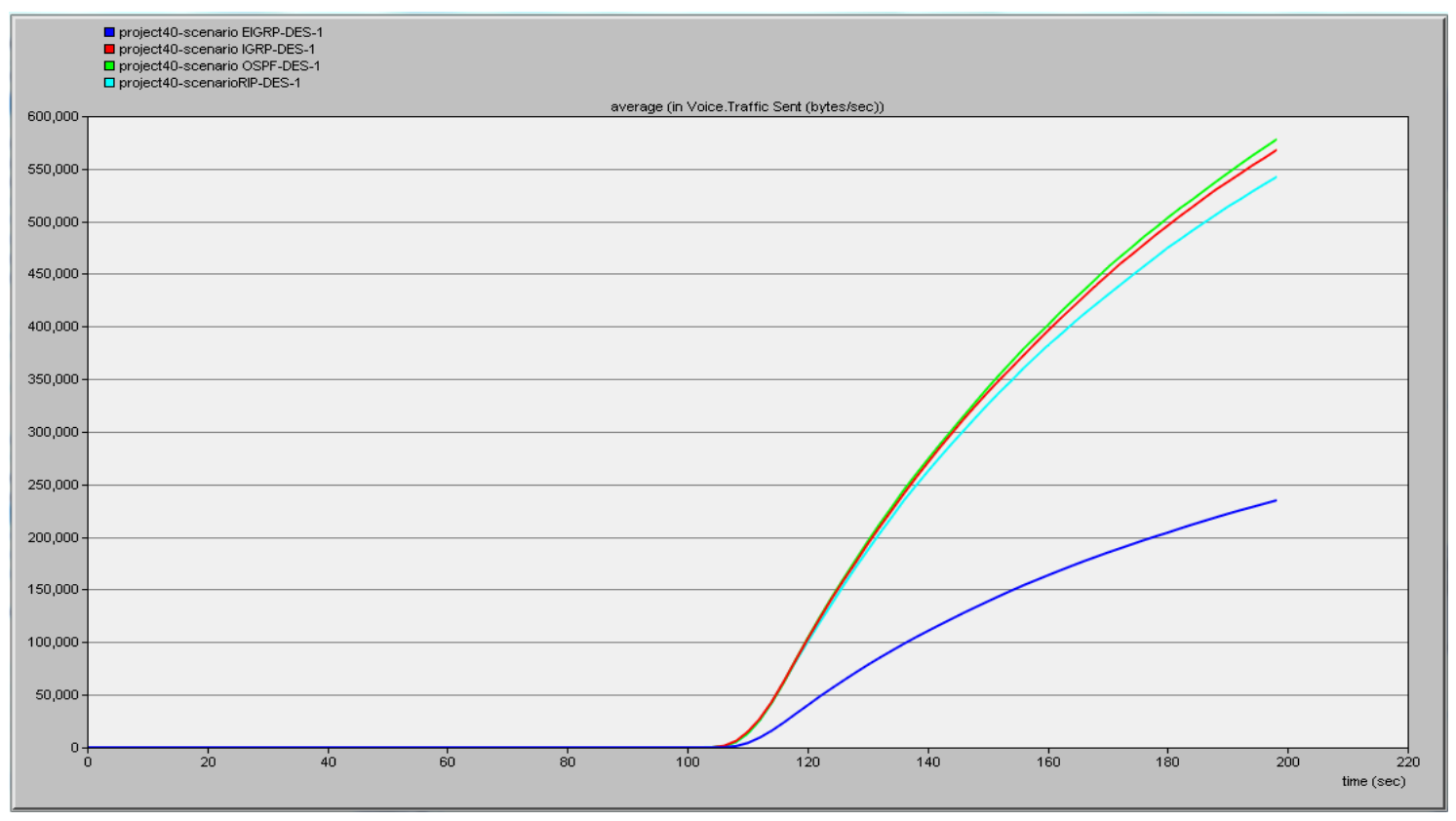

Fig.9. Shown average voice traffic sent (byte on a sec )

Figure 9 shows the variations of the voice traffic sent using four protocols. It has been seen that OSPF archives a high level of average compared to the EIGRP at the lower level. This confirmes that the protocol efficiency of OSPF is better than the one achieved by EIGRP based on traffic sent data.

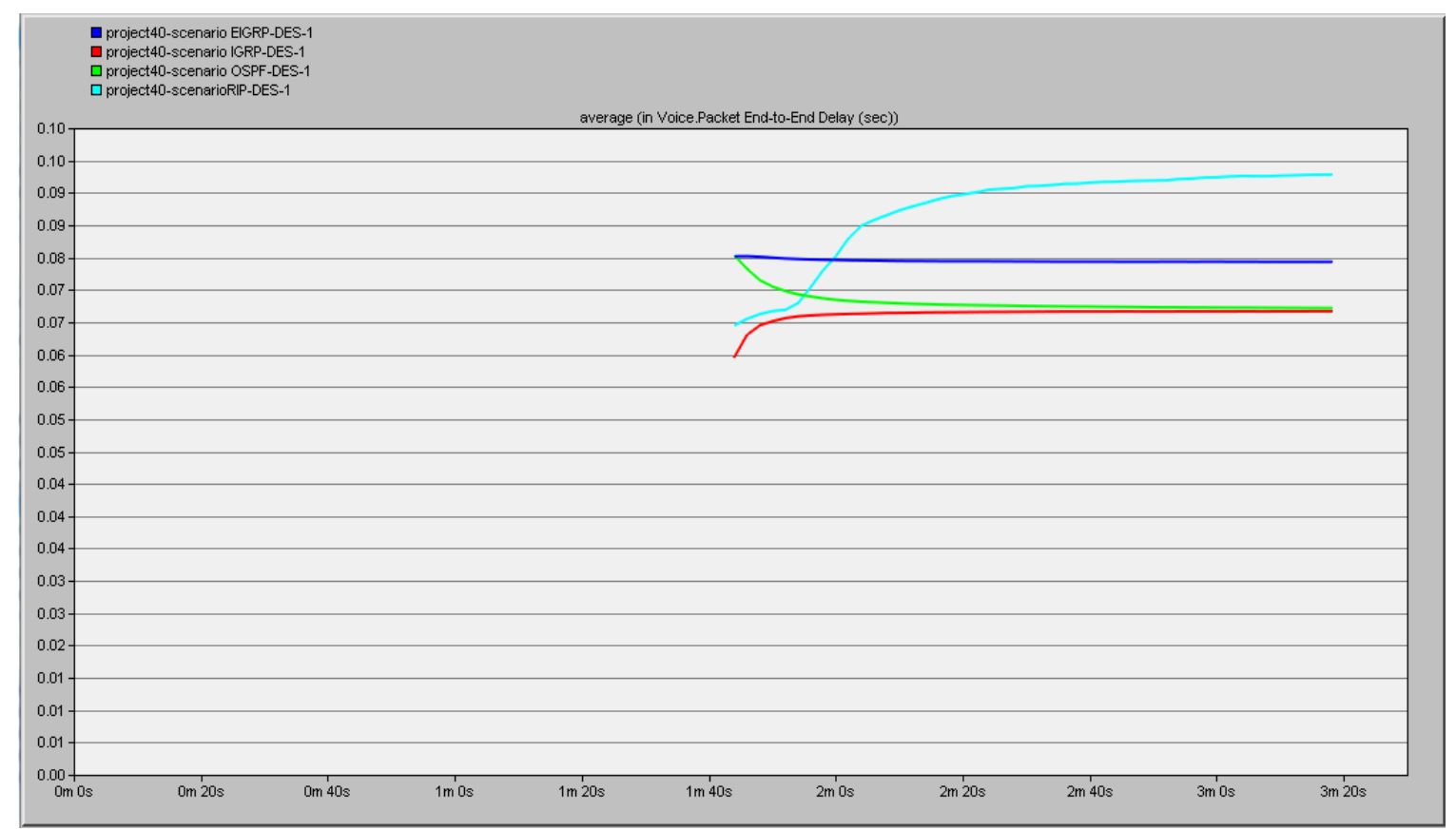

Fig.10. Average (voice) packet end-to-end delay by sec with $300 \mathrm{sec}$ 
From this Simulation that protocols RIP, OSPF, IGRIP, EIGRP levels delay packet in used application voice represent the lowest and highest delays, respectively, that protocol RIP has the highest delay and the protocol IGRP have the shortest delay as shown in Figure 10.

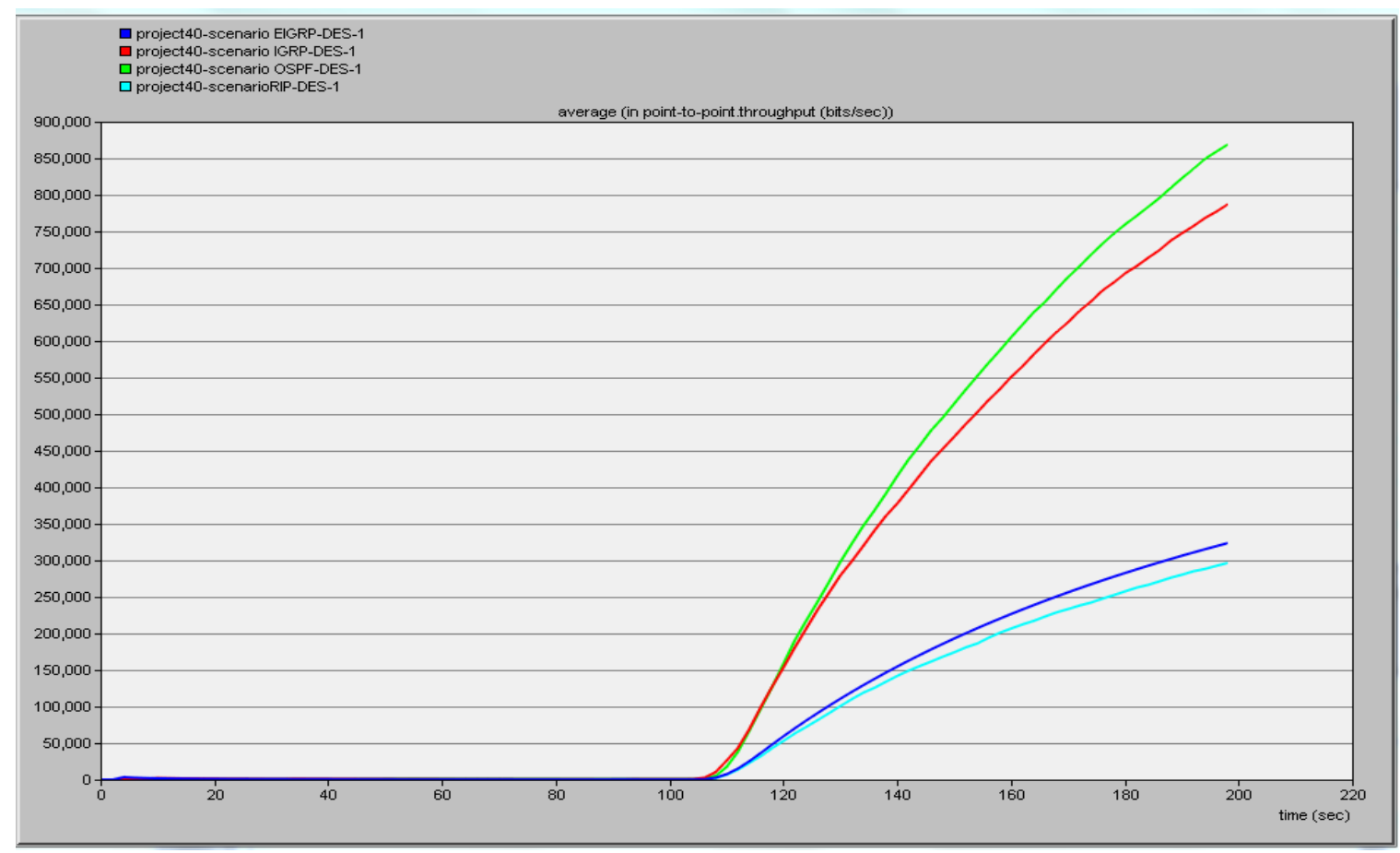

Fig.11. Rate point to point throughput (bit/sec)

Figure 11 shows the average throughput between point to point on the network over four protocols. It is clear that a higher throughput achieved by the OSPF protocols compared to all other protocols considered in this test example.

\section{Conclusion}

In this paper, EIGRP, OSPF, RIP and IGRP protocols have been studied and investigated through extensive simulation process by carefully selective parameters to achieve their properties in terms of their routing algorithms. The parameters involved here are voice traffic sent, voice traffic received, average end to end delay and average point to point throughput using OPNET 14.5 software. It was found that OSPF outperforms the other protocols based on certain parameters that define the network level.

\section{Refernces}

[1]Jagdeep Singh, Rajiv Mahajan, "Simulation Based comparitive study of RIP OSPF and EIGRP", International Journal of Advanced Research in Computer Science and Software Engineering, vol. 3, no. 8, pp. 1-4, August 2013.

[2]Vetriselvan. V, Mahendran. M, "Survey on RIP OSPF and EIGRP Routing protocols", International Journal of Computer Science and Information Technology, vol. 5, no. 2, pp. 2-7, 2014.

[3] Verma, Abhishek, and Neha Bhardwaj. "A review on routing information protocol (RIP) and open shortest path first (OSPF) routing protocol." International Journal of Future Generation Communication and Networking 9.4 (2016): 161-170.

[4]Fiţigău, Ioan, and Gavril Toderean. "Network performance evaluation for RIP, OSPF and EIGRP routing protocols." Proceedings of the International Conference on ELECTRONICS, COMPUTERS and ARTIFICIAL INTELLIGENCE-ECAI-2013. IEEE, 2013.

[5]Roy, Sudipta, et al. "International Journal of Advanced Research in Computer Science and Software Engineering." International Journal 3.6 (2013).

[6]Xu, Don, and Ljiljana Trajkovic. "Performance analysis of RIP, EIGRP, and OSPF using OPNET." (2011).

[7] W. Gajanan, K. Anne,: "Performance analysis of routing protocols in MANET". Indonesian Journal of Electrical Engineering and Computer Science.Vol. 17, No. 2, 2020 
[8]Farhangi, S., A. Rostami, and S. Golmohammadi. "Performance Comparison of mixed protocols based on EIGRP, IS-IS and OSPF for real-time applications." Middle-East Journal of Scientific Research 12.11 (2012): 1502-1508.

[9]Narula, Rajneesh, and Pallavi Aggarwal. "Performance Evaluation of RIP and OSPF in IPv6 using OPNET 14.5 Simulator." International journal of technical research and applications 2.6 (2014): 37-41.

[10] S. G. Thornier, "Dynamic Routing protocol implementation decision between EIGRP OSPF and RIP based in technical background using OPNET Modeler", in Proc. Second International Conference on Computer and Network Technology(I CCNT), pp. 191-195, 2010.

[11] IKram Ud Din, Saeed Mahfooz, Muhammad Adnan, "Anelysis of the Routing Protocols in Real Time Transmission: A Comparitive Study", Global Journal of Computer Science and Technology, vol. 4, no. 5, pp. 4-6, 2010.

[12] B. Fortz, M. Thorup, "Optimizing OSPF/IS-IS weights in a changing world", IEEE Journal on Selected Areas in Communications 2002, vol. 20, no. 4, pp. 75-80, 2002.

[13] M. Kang, and Y. Chung, "An Improved Hybrid Routing Protocol Combining MANET and DTN". Electronics 2020, 9, 439; doi:10.3390/electronics9030439. 2020 